\title{
SEQUENTIAL CONFIDENCE INTERVALS FOR A POPULATION SIZE WITH FIXED PROPORTIONAL ACCURACY
}

\author{
Ki Heon Choi, ${ }^{1}$ Ruby C. Weng, ${ }^{2}$ and Michael Woodroofe ${ }^{3}$ \\ ${ }^{1}$ Duksung Women's University, \\ Seoul, Korea, 132-714 \\ ${ }^{2}$ National Chengchi University, \\ Taipei, Taiwan, 11623 \\ ${ }^{3}$ University of Michigan, Ann Arbor, \\ MI 48109-1027, USA
}

\begin{abstract}
Consider a population of hidden objects of which the total number $N$ is unknown. A search for the objects may be conducted in which the times at which the objects were found is recorded, along with the total number of objects found. From such data, a confidence interval for $N$ is desired with specified proportional accuracy. Two sequential sampling plans are proposed for this problem, for two sets of underlying assumptions, and their properties are studied through asymptotic analysis and simulation.

Key Words: Brownian motion; Convergence in distribution; Exponential distributions; Hidden objects; Order statistics; Poisson processes; Simulation; Stopping times.
\end{abstract}




\section{INTRODUCTION}

Consider the problem of searching for hidden objects - for example, diseased individuals, donors to a charitable organization, fish in a lake, or potential voters in a voter registration drive. Let $N$ denote the total number of objects, and suppose that $N$ is unknown, but all objects would be found if the search were continued indefinitely. This problem has been considered by several authors, including Starr [8], Vardi [9], Kramer and Starr [7], Bellout [1], and Dalal and Mallows [5]. The emphasis in the latter work was finding optimal searching policies, stopping times that maximize an expected reward, like the number of objects found, less a cost of sampling. Here another aspect of the problem is considered, finding a confidence interval for $N$ with fixed proportional accuracy that is valid for large $N$, at least.

Some notation is required to describe our results. Imagine labels painted on the objects and let $\tilde{T}_{i}$ denote the time at which the object labeled $i$ would be found if the search were continued indefinitely. We suppose throughout that $\tilde{T}, \cdots, \tilde{T}_{N} \sim^{\text {ind }} F$ are independent and identically distributed with a continuous distribution function $F$ for which $F(0)=0$. The distribution function $F$ may depend on an unknown parameter $\theta$, or not. Let $T_{1} \leq \cdots \leq T_{N}$ denote the order statistics $\tilde{T}, \cdots, \tilde{T}_{N}$. If the search is continued for $t$ units of times, then the available data consists of the number of objects found and the times at which they were found; in symbols,

$$
K_{t}=\#\left\{i \leq N: \tilde{T}_{i}^{\sim} \leq t\right\} \text { and } 0 \leq T_{1} \leq \cdots \leq T_{K_{t}} .
$$

Let $\hat{N}_{t}$ denote an estimator of $N$, based on this data-for example, $\hat{N}_{t}=K_{t} / F(t)$, if $F$ is known. Then, stopping times $\tau_{h}$ are sought for which

$$
\lim _{h \downarrow 0} \lim _{N \rightarrow \infty} P_{\theta, N}\left\{\left|\hat{N}_{\tau_{h}}-N\right| \leq h \hat{N}_{\tau_{h}}\right\}=\gamma,
$$

for all values of the unknown parameter $\theta$, if present, where $\gamma$ is the desired confidence level and $P_{\theta, N}$ denotes probability in the model described above.

Relation (2) is established twice, in Section 2 under the assumption that $F$ is a known, and again in Sections 3 and 5 under the assumption that $F$ is exponential with unknown failure rate $\theta$. In fact, $\lim _{N \rightarrow \infty}$ $P_{\theta, N}\left\{\left|\hat{N}_{\tau_{h}}-N\right| \leq h \hat{N}_{\tau_{h}}\right\}$, is computed for fixed $h>0$ in both cases. As will be apparent, the case of unknown $\theta$ is much more difficult from both mathematical and inferential viewpoints. The results of a simulation study are presented in Section 4, and the proof of the main result in Section 5. 


\section{KNOWN $F$}

In this section, $F$ is assumed to be a known, continuous distribution function that is strictly increasing on the interval $\left(0, b_{F}\right)$, where $b_{F}=$ $\sup \{t: F(t)<1\} \leq \infty$. Then the maximum likelihood estimator of $F$ after $t$ time units of observation is (an integer adjacent to)

$$
\hat{N}_{t}=\frac{K_{t}}{F(t)}
$$

for $0<t<b_{F}$. Since $K_{t}$ has a binomial distribution, the mean and variance of $\hat{N}_{t}$ are $E_{N}\left[\hat{N}_{t}\right]=N$ and $D_{N}^{2}\left[\hat{N}_{t}\right]=N \sigma^{2}(t)$, where

$$
\sigma^{2}(t)=\frac{1}{F(t)}-1
$$

and $\hat{N}_{t}$ is asymptotically normal as $N \rightarrow \infty$ for fixed $t>0$; that is,

$$
\frac{\hat{N}_{t}-N}{\sqrt{N \sigma^{2}(t)}} \Rightarrow Z \sim \Phi,
$$

where $\Rightarrow$ denotes convergence in distribution and $\Phi$ denotes the standard normal distribution function. In fact, (5) holds for sequences $t=t_{N}>0$ for which $N \sigma^{2}\left(t_{N}\right) \rightarrow \infty$ as $N \rightarrow \infty$. Using asymptotic normality to set an approximate confidence interval and imposing the condition that the half width of the interval be at most $h \hat{N}_{t}$, as in Chow and Robbins [3], suggests sampling until $\hat{N}_{t} \geq z^{2} \sigma^{2}(t) / h^{2}$, where $\Phi(z)=(1+\gamma) / 2$ (and $\gamma$ is the desired confidence coefficient). Using (3) and (4) this may be written as the stopping time

$$
\tau_{h}=\inf \left\{t>0: K_{t}>\frac{z^{2}}{h^{2}}[1-F(t)]\right\} .
$$

In the theorem below, $L_{s}, 0 \leq s<\infty$, denotes a homogeneous Poisson process with mean $E\left(L_{s}\right)=s$. Further, let $m=m(z, h)=\left[z^{2} / h^{2}\right]$, the least integer that is greater than or equal to $z^{2} / h^{2}$, and let

$$
\eta_{h}=\inf \left\{s>0: L_{s} \geq m\right\}
$$

Theorem 2.1 If $\hat{N}_{t}$ and $\tau_{h}$ are defined by (3) and (6), then $N F\left(\tau_{h}\right) \Rightarrow \eta_{h}$, and

$$
\lim _{N \rightarrow \infty} P_{N}\left\{\left|\hat{N}_{\tau_{h}}-N\right| \leq h \hat{N}_{\tau_{h}}\right\}=P\left\{\left|\eta_{h}-m\right| \leq h m\right\}
$$

for each fixed $h>0$. 
Proof. Let $s$ and $t$ be related by $N F(t)=s$, and let $H_{N}(s)=K_{t}$; that is,

$$
H_{N}(s)=K\left[F^{-1}\left(\frac{s}{N}\right)\right]
$$

for $0 \leq s \leq N b_{F}$, where $K(u)$ is written for $K_{u}$ when $u$ is a complicated expression. Next, let $D[0, c]$ denote the space of càdlàg functions on the interval [0,c], as described by Billingsley [2, Ch.3], for example. Then it is easily seen that $H_{N} \Rightarrow L$ in $D[0, \infty)$; that is, the restriction of $H_{N}$ to $[0, c]$ converges to the restriction of $L$ in $D[0, c]$ for all $0<c<\infty$. It follows that

$$
N F\left(\tau_{h}\right)=\inf \left\{s>0: H_{N}(s)>\frac{z^{2}}{h^{2}}\left(1-\frac{s}{N}\right)\right\} \Rightarrow \eta_{h}
$$

as $N \rightarrow \infty$. Next, since $\left|\hat{N}_{t}-N\right| \leq h \hat{N}_{t}$ iff $\left|K_{t}-N F(t)\right| \leq h K t$, and since $L_{\eta_{h}}=m w \cdot p \cdot 1$,

$$
\begin{aligned}
P_{N}\left\{\left|\hat{N}_{\tau_{h}}-N\right| \leq h \hat{N}_{\tau_{h}}\right\} & =P_{N}\left\{\left|K_{\tau_{h}}-N F\left(\tau_{h}\right)\right| \leq h K_{\tau_{h}}\right\} \\
& \rightarrow P\left\{\left|\eta_{h}-m\right| \leq h m\right\}
\end{aligned}
$$

as $N \rightarrow \infty$, establishing (7).

The following easily verified inequality is used in the proof of the next theorem. If $X$ has a binomial distribution with parameters $n$ and $p>0$, then

$$
E\left\{\frac{1}{(X+1)(X+2)}\right\} \leq \frac{1}{n^{2} p^{2}}
$$

Theorem 2.2 Relation (2) holds,

$$
\lim _{h \downarrow 0} \lim _{N \rightarrow \infty} E_{N}\left|h^{2} N F\left(\tau_{h}\right)-z^{2}\right|=0 .
$$

and

$$
\lim _{h \downarrow 0} \lim _{N \rightarrow \infty} E_{N}\left\{\frac{\hat{N}_{\tau_{h}}}{N}\right\}=1 .
$$

Proof. Relation (2) follows easily from (7), since $\eta_{h}$ is the sum of $m$ i.i.d. exponentially distributed random variables, $m \rightarrow \infty$, and $h m \sim z \sqrt{m}$ as $h \downarrow 0$. Thus,

$$
\lim _{h \downarrow 0} P\left\{\left|\eta_{h}-m\right| \leq h m\right\}=\lim _{h \downarrow 0} P\left\{\left|\frac{\eta_{h}-m}{\sqrt{m}}\right| \leq h \sqrt{m}\right\}=2 \Phi(z)-1=\gamma .
$$


Similarly, using (8) and the structure of $\eta_{h}$,

$$
\lim _{h \downarrow 0} \lim _{N \rightarrow \infty} P_{N}\left\{\left|h^{2} N F\left(\tau_{h}\right)-z^{2}\right|>\epsilon\right\}=\lim _{h \downarrow 0} P\left\{\left|h^{2} \eta_{h}-z^{2}\right|>\epsilon\right\}=0
$$

for each $\epsilon>0$. To see that $h^{2} N F\left(\tau_{h}\right)$ are uniformly integrable, first observe that

$$
\begin{aligned}
P_{N}\left\{\tau_{h}>t\right\} & \leq P_{N}\left\{K_{t} \leq \frac{z^{2}}{h^{2}}\right\} \\
& \leq P\left\{\frac{1}{\left(K_{t}+1\right)\left(K_{t}+2\right)}>\frac{h^{4}}{\left(z^{2}+2 h^{2}\right)^{2}}\right\} \\
& \leq \frac{\left(z^{2}+2 h^{2}\right)^{2}}{h^{4}} \frac{1}{N^{2} F(t)^{2}}
\end{aligned}
$$

for all $N, h$, and $t$. Thus, setting $t=F^{-1}\left(s / h^{2} N\right)$,

$$
P_{N}\left\{h^{2} N F\left(\tau_{h}\right)>s\right\} \leq \frac{\left(z^{2}+2 h^{2}\right)^{2}}{s^{2}}
$$

for all $N, h$, and $s$, from which uniform integrability and (9) follow.

The proof of (10) is similar. From Theorem 2.1, $\hat{N}_{\tau_{h}} / N=K\left(\tau_{h}\right) /$ $N F\left(\tau_{h}\right) \Rightarrow m / \eta_{h}$ as $N \rightarrow \infty$ for fixed $h>0$. It is shown below that $\hat{N}_{\tau_{h}} / N$ is uniformly integrable in $N$ for sufficiently small $h>0$, and it then follows that

$$
\lim _{N \rightarrow \infty} E_{N}\left\{\frac{\hat{N}_{\tau_{h}}}{N}\right\}=E\left\{\frac{m}{\eta_{h}}\right\}=\frac{m}{m-1}
$$

which approaches 1 as $h \rightarrow 0$.

To establish uniform integrability, let $h$ be so small that $z^{2} / h^{2}>1$, and let $\epsilon>0$ be so small that $\left(z^{2} / h^{2}\right)(1-\epsilon)>1$. Then $K\left(\tau_{h}\right) \geq 2$ when $\tau_{h} \leq \epsilon$ and, therefore,

$$
\frac{\hat{N}_{\tau_{h}}}{N}=\frac{K\left(\tau_{h}\right)}{N F\left(\tau_{h}\right)} \leq \frac{2}{N F(\epsilon)}+\sup _{t \geq T_{2}} \frac{K_{t}}{N F(t)}
$$

The first term on the right side approaches zero. For the second,

$$
\sup _{t \geq T_{2}} \frac{K_{t}}{N F(t)}=\max _{2 \leq k \leq N} \frac{k}{N F\left(T_{k}\right)} .
$$


Here $F\left(T_{k}\right), k=1, \cdots, N$, have the same joint distribution as $S_{k} / S_{N+1}$, where $S_{k}=E_{1}+\cdots+E_{k}$ are sums of i.i.d. standard exponential random variables. Let $1<p, q<\infty$ be conjugate values; that is $1 / p+1 / q=1$. If $\alpha<2$, then there is, a $p$ for which $q \alpha<2$, in which case,

$$
E\left\{\left[\max _{2 \leq k \leq N} \frac{k}{N F\left(T_{k}\right)}\right]^{\alpha}\right\} \leq E\left\{\left[\max _{k \geq 2} \frac{k}{S_{k}}\right]^{q \alpha}\right\}^{\frac{1}{q}} \times E\left\{\left[\frac{S_{N+1}}{N}\right]^{p \alpha}\right\}^{\frac{1}{p}} .
$$

The first factor is finite, since $S_{k} / k$ is a reverse martingale; and the second is bounded in $N$. Uniform integrability and (10) follow.

If the procedure is modified slightly, then it is possible to make the limit of the coverage probabilities exceed $\gamma$ for all sufficiently small $h>0$. Given a design parameter $b>0$, let

$$
\tau_{h}^{*}=\inf \left\{t>0: K_{t}>\left(\frac{z^{2}}{h^{2}}+b\right)[1-F(t)]\right\} .
$$

Theorem 2.3 If $b>\left(2 z^{4}-11 z^{2}+3\right) / 18$, then $\lim _{N \rightarrow \infty} P_{N} \quad\left\{\left|\hat{N}_{\tau_{h}}-N\right| \leq\right.$ $\left.h \hat{N}_{\tau_{h}}\right\}>\gamma$ for all sufficiently small $h>0$.

Proof. Let $m^{*}$ be the least integer that is greater than or equal to $h^{-2} z^{2}+b$. Then, as in the proof of Theorem 2.1, it may be shown that (8) holds with $\tau_{h}, m$, and $\eta_{h}$ replaced by $\tau_{h}^{*}, m^{*}$, and $\eta_{h}^{*}=\inf \left\{s>0: L_{s} \geq m^{*}\right\}$. Here $\eta_{h}^{*}$ is again the sum of independent standard exponential random variables, and an Edgeworth expansion leads to

$$
\begin{aligned}
P\left\{\left|\eta_{h}^{*}-m^{*}\right| \leq h m^{*}\right\}= & 2 \Phi\left(h \sqrt{m^{*}}\right)-1+\frac{1}{2 m^{*}} \varphi^{\prime \prime \prime}(h \sqrt{m *}) \\
& +\frac{1}{9 m^{*}} \varphi^{(v)}\left(h \sqrt{m^{*}}\right)+o\left(h^{2}\right) \\
= & 2 \Phi(z)-1+\frac{b z}{m} \varphi(z)-\frac{2 z^{5}-11 z^{3}+3 z}{18 m} \varphi(z)+o\left(h^{2}\right)
\end{aligned}
$$

as $h \downarrow 0$, where $\varphi=\Phi^{\prime}$ is the standard normal density, and the right side exceeds $\gamma$ for sufficiently small $h>0$ if $b>\left(2 z^{4}-11 z^{2}+3\right) / 18$.

\section{UNKNOWN $F$}

In this section $F=F_{\theta}$ is assumed to be an exponential distribution with unknown failure rate $\theta$; that is, $F_{\theta}(x)=1-e^{-\theta x}$ for $x \geq 0$. There are then two aspects to the problem, estimating $\theta$ and estimating $N$. 
Estimating $\theta$. It is convenient to begin with the likelihood function: if $k \geq 1$ and $0 \leq t_{1} \leq \cdots \leq t_{k} \leq t$, then

$$
\begin{aligned}
P_{\theta, N}\left\{K_{t}=\right. & \left.k, t_{1} \leq T_{1} \leq t_{1}+d t_{1}, \cdots, t_{k} \leq T_{k} \leq t_{k}+d t_{k}\right\} \\
= & (N)_{k} e^{-(N-k) \theta t} \theta e^{-\theta t_{1}} d t_{1} \times \cdots \times \theta e^{-\theta t_{k}} d t_{k} \\
& \propto(N)_{k} \theta^{k} \exp \left\{-(N-k) \theta t-\theta s_{t}\right\} d t_{1} \cdots d t_{k},
\end{aligned}
$$

where $(N)_{k}=N(N-1) \times \cdots \times(N-k+1)$ and $s_{t}=t_{1}+\cdots+t_{k}$. Recalling that $K_{t}$ has a binomial distribution, it is then easily seen that the conditional density of $X_{j}=T_{j} / t, j=1, \cdots, k$, given that $K_{t}=k$, is the same as the distribution of the order statistics of a sample of size $k$ from the density

$$
f_{\theta t}(x)=\frac{\theta t e^{-\theta t x}}{1-e^{-\theta t}}, \quad 0 \leq x \leq 1 .
$$

In particular, the conditional distribution depends only on $\theta t$ and not on $N$. This provides a method for estimating $\theta$.

The family of densities $f_{\omega}$, implicityly defined by (11), is an exponential family, $f_{\omega}(x)=\exp [-\omega x-\psi(\omega)], 0 \leq x \leq 1$, with cumulant generating function

$$
\psi(\omega)=\log \left(\frac{1-e^{-\omega}}{\omega}\right) .
$$

The natural parameter space for this family is $-\infty<\omega<\infty$, however, not $0<\omega<\infty$. So, (conditional) maximum likelihood estimators may be obtained from the method of moments, but the formal expressions for these may give negative estimators. To circumvent the problem of negative estimators and also the less obvious problem of occasional large estimates, consider penalized (conditional) maximum likelihood estimators. For given values of $\alpha, \beta>0$, let $\ell^{*}(\theta \mid t)$ be the logarithm of the conditional likelihood given $K_{t}$ times $\theta^{\alpha} e^{-\beta \theta}$. Then

$$
\ell^{*}(\theta \mid t)=-\theta\left[\beta+S_{t}\right]+\left(K_{t}+\alpha\right) \log (\theta)-K_{t} \log \left(1-e^{-\theta t}\right)+C,
$$

where

$$
S_{t}=T_{1}+\cdots+T_{K_{t}}
$$

and $C$ does not depend on $\theta$. Then $\ell^{*}(\theta \mid t)$ attains its maximum at a unique point $\hat{\theta}_{t}$ for which $\partial \ell^{*}(\theta \mid t) / \partial \theta=0$, or equivalently,

$$
\frac{K_{t}+\alpha}{\hat{\theta}_{t}}-\frac{t K_{t}}{e^{t \hat{\theta}_{t}}-1}=\beta+S_{t} .
$$


Equation (12) is called the penalized likelihood equation below, and $\hat{\theta}_{t}$ the penalized maximum likelihood estimator. Of course, $\hat{\theta}_{t}$ is also the posterior mode for a prior proportional to $\theta^{\alpha} e^{-\beta \theta}$, and this connection may be useful in specifying values of $\alpha$ and $\beta$.

Lemma 3.1 For fixed $\theta>0$ and $t>0$, the conditional distribution of $t \hat{\theta}_{t}$ given $K_{t}=k$ is asymptotically normal with mean $t \theta$ and variance $1 / k \psi^{\prime \prime}(t \theta)$ as $k \rightarrow \infty$. As $N \rightarrow \infty$ the unconditional distribution is asymptotically normal with mean $t \theta$ and variance $1 /\left[N\left(1-e^{-t \theta}\right) \psi^{\prime \prime}(t \theta)\right]$.

Proof. Without the penalty term, these assertions follow immediately from the asymptotic properties of maximum likelihood estimators and the Law of Large Numbers for $K_{t}$; and it is not difficult to see that the penalty terms do not affect the asymptotic distributions here (though they do in Proposition 5.2 below). The details are omitted, because the lemma is only used to motivate the stopping rule below.

Estimating $N$. With $\hat{\theta}_{t}$ as in (12), now let

$$
\hat{N}_{t}=\frac{K_{t}}{1-e^{-t \hat{\theta}_{t}}} .
$$

Lemma 3.2 For fixed $\theta, t>0, \hat{N}_{t}$ is asymptotically normal with mean $N$ and variance $N \sigma^{2}(t \theta)$ as $N \rightarrow \infty$, where

$$
\sigma^{2}(\omega)=\left(\frac{1}{e^{\omega}-1}\right)\left[\frac{e^{\omega}}{\left(e^{\omega}-1\right)^{2} \psi^{\prime \prime}(\omega)}+1\right]
$$

Proof. Lemma 3.2 follows from Lemma 3.1 and the asymptotic normality of $K_{t}$ by writing

$$
\begin{aligned}
\hat{N}_{t}-N & =\frac{K_{t}}{1-e^{-t \hat{\theta}_{t}}}-N \frac{1-e^{-t \hat{\theta}_{t}}}{1-e^{-t \hat{\theta}_{t}}} \\
& =\left(\frac{1}{1-e^{-t \hat{\theta}_{t}}}\right)\left[K_{t}-N\left(1-e^{-t \theta}\right)+N\left(e^{-t \hat{\theta}_{t}}-e^{-t \theta}\right)\right],
\end{aligned}
$$

and using the delta method. The details are omitted, because the lemma is only used to motivate the stopping rule below.

As in the case of known $F$, the asymptotic distribution of $N$ suggests that sampling be continued until $\hat{N}_{t} \geq z^{2} \sigma^{2}\left(t \hat{\theta}_{t}\right) / h^{2}$. Multiplying this inequality by $\left(1-e^{-t \hat{\theta}_{t}}\right)$ then leads to the stopping time

$$
\tau_{h}=\inf \left\{t>0: K_{t} \geq \frac{z^{2}}{h^{2}} \rho\left(t \hat{\theta}_{t}\right)\right\},
$$


where

$$
\rho(\omega)=\left(1-e^{-\omega}\right) \sigma^{2}(\omega)=\frac{\omega^{2}}{\left(e^{\omega}-1\right)^{2}-\omega^{2} e^{\omega}}+e^{-\omega} .
$$

Then $\tau_{h}<\infty$ w.p.1 for each fixed $N \geq 1, \theta>0$, and $h>0$. To see this, first observe that the left side of (12) is at least $\alpha / \hat{\theta}_{t}$, that the right side remains bounded as $t \rightarrow \infty$ for fixed $N$ and $h$ and, therefore, that $\lim _{t \rightarrow \infty} t \hat{\theta}_{t}=\infty$ w.p.1. That $\tau_{h}<\infty$ w.p.1 then follows since $\rho(\omega) \rightarrow 0$ as $\omega \rightarrow \infty$.

The proposed confidence interval is $\hat{N}_{\tau_{h}} \pm h \hat{N}_{\tau_{h}}$.

Theorem 3.4 With $\hat{N}_{t}$ and $\tau_{h}$ defined by (13) and (14), (2) holds and

$$
\lim _{h \downarrow 0} \lim _{N \rightarrow \infty} E_{\theta, N}\left|h^{\frac{2}{3}} N^{\frac{1}{3}} F_{\theta}\left(\tau_{h}\right)-\left(12 z^{2}\right)^{\frac{1}{3}}\right|=0
$$

for all $0<\theta<\infty$.

The proof uses weak convergence of processes, as in the proof of Theorem 2.1, but the scaling is different and implementation is more complicated. The proof is presented in Section 5.

\section{SIMULATIONS}

The actual coverage probability, expected stopping time, and the expected value of the estimated population size are assessed through simulation in Tables I, II and III below for $\gamma=2 \Phi(2)-1 \approx .954$, and selected values of $\alpha, \beta, h$, and $N$. In all tables, $F$ is the standard exponential distribution.

The procedure for known $F$ is studied in Table I, and that for unknown $\theta$ is in Tables II and III. In Table I, the simulated coverage

Table I. $N=2,500, \theta$ Known

\begin{tabular}{lcccr}
\hline & $h=.1$ & $h=.2$ & $h=.3$ & $h=.4$ \\
\hline prob & .955 & .951 & .945 & .937 \\
$\mathrm{E}_{\theta, N \tau h}$ & .148 & .039 & .017 & .010 \\
$\mathrm{E}_{\theta, N}\left(N_{\tau h}\right)$ & 2505 & 2523 & 2553 & 2603 \\
\hline
\end{tabular}

Monte Carlo estimates based on 10,000 replications; prob $=P_{\theta, N}\left\{\left|\hat{N}_{\tau_{h}}-N\right| \leq h \hat{N}_{\tau_{h}}\right\}$, where $\tau_{h}$ is defined as in (6) for known $F$ and as in (13) for unknown $F$; both with $100 \gamma \%$ confidence, $\gamma=2 \Phi(z)-1, z=2 ; E_{\tau_{h}}, E \hat{N}_{\tau_{h}}$, and $E \hat{\theta}$ are the estimated mean values of $\tau_{h}$ and $\hat{\theta}$ over 10,000 replications. 
Table II. $N=2,500, h=.1, \theta$ Unknown

\begin{tabular}{|c|c|c|c|c|c|c|c|c|}
\hline \multirow[b]{2}{*}{$\beta$} & \multicolumn{4}{|c|}{$\alpha=.01$} & \multicolumn{4}{|c|}{$\alpha=.25$} \\
\hline & prob & $E_{\theta, N \tau h}$ & $E_{\theta, N} \hat{\theta}$ & $E_{\theta, N}\left(\hat{N}_{\tau h}\right)$ & prob & $E_{\theta, N \tau h}$ & $E_{\theta, N} \hat{\theta}$ & $E_{\theta, N}\left(\hat{N}_{\tau h}\right)$ \\
\hline .0 & .949 & 1.044 & 1.014 & 2484 & .949 & 1.042 & 1.017 & 2481 \\
\hline .01 & .948 & 1.044 & 1.015 & 2484 & .949 & 1.042 & 1.017 & 2481 \\
\hline .05 & .950 & 1.045 & 1.014 & 2485 & .951 & 1.043 & 1.016 & 2482 \\
\hline .1 & .950 & 1.045 & 1.014 & 2485 & .951 & 1.043 & 1.016 & 2482 \\
\hline .5 & .953 & 1.048 & 1.010 & 2490 & .953 & 1.046 & 1.012 & 2488 \\
\hline
\end{tabular}

Monte Carlo estimates based on 10,000 replications; prob $=P_{\theta, N}\left\{\left|\hat{N}_{\tau_{h}}-N\right| \leq h \hat{N}_{\tau_{h}}\right\}$, where $\tau_{h}$ is defined as in (6) for known $F$ and as in (13) for unknown $F$; both with $100 \gamma \%$ confidence, $\gamma=2 \Phi(z)-1, z=2 ; E_{\tau_{h}}, E \hat{N}_{\tau_{h}}$, and $E \hat{\theta}$ are the estimated mean values of $\tau_{h}$ and $\hat{\theta}$ over 10,000 replications.

Table III. $\quad N=1,000$ and 5,000, $\theta$ Unknown

\begin{tabular}{|c|c|c|c|c|c|c|c|c|}
\hline \multicolumn{9}{|c|}{$N=1,000$} \\
\hline \multirow[b]{2}{*}{$h$} & \multicolumn{4}{|c|}{$\alpha=.01, \beta=.5$} & \multicolumn{4}{|c|}{$\alpha=.01, \beta=.5$} \\
\hline & prob & $E_{\theta, N \tau h}$ & $E_{\theta, N} \hat{\theta}$ & $E_{\theta, N}\left(\hat{N}_{\tau h}\right)$ & prob & $E_{\theta, N \tau h}$ & $E_{\theta, N} \hat{\theta}$ & $E_{\theta, N}\left(\hat{N}_{\tau h}\right)$ \\
\hline .1 & .954 & 1.361 & 1.013 & 995 & .952 & 1.358 & 1.015 & 994 \\
\hline .2 & .953 & .915 & 1.039 & 987 & .954 & .914 & 1.041 & 985 \\
\hline \multicolumn{9}{|c|}{$N=5,000$} \\
\hline & \multicolumn{4}{|c|}{$\alpha=.01, \beta=.5$} & \multicolumn{4}{|c|}{$\alpha=.01, \beta=.5$} \\
\hline$h$ & prob & $E_{\theta, N \tau h}$ & $E_{\theta, N} \hat{\theta}$ & $E_{\theta, N}\left(\hat{N}_{\tau h}\right)$ & prob & $E_{\theta, N \tau h}$ & $E_{\theta, N} \hat{\theta}$ & $E_{\theta, N}\left(\hat{N}_{\tau h}\right)$ \\
\hline .1 & .955 & .856 & 1.008 & 4984 & .955 & .856 & 1.009 & 4982 \\
\hline .2 & .953 & .562 & 1.031 & 4939 & .954 & .561 & 1.033 & 4933 \\
\hline
\end{tabular}

Monte Carlo estimates based on 10,000 replications; prob $=P_{\theta, N}\left\{\left|\hat{N}_{\tau_{h}}-N\right| \leq h \hat{N}_{\tau_{h}}\right\}$, where $\tau_{h}$ is defined as in (6) for known $F$ and as in (13) for unknown $F$; both with $100 \gamma \%$ confidence, $\gamma=2 \Phi(z)-1, z=2 ; E_{\tau_{h}}, E \hat{N}_{\tau_{h}}$, and $E \hat{\theta}$ are the estimated mean values of $\tau_{h}$ and $\hat{\theta}$ over 10,000 replications.

probabilities agree well with the nominal value for $h=.1$ and .2 . The lack of agreement for large $h$ is not surprising. The dependence of the coverage probabilities and expected sample size on $\alpha$ and $\beta$ in Table II is interesting. Both are fairly insensitive to the selected $\alpha$ and $\beta$. The coverage probabilities for $\beta=.0$ and .01 are significantly different from the nominal value .954 at significance level 0.05 , but just barely so; those for $\beta=.05, .1$, and .5 
agree with the nominal value .954 . The other notable feature of Table II is that $E\left(\tau_{h}\right)$ is much larger, reflecting the greater difficulty of the problem when $F$ is unknown. We select $(\alpha, \beta)=(.01, .5)$ and $(.1, .5)$ for further study. Table III reports the simulated results with choices of $N$ and $h$. None of the simulated coverage probabilities is significantly different from 0.954 at significance level 0.05 in Table III.

\section{PROOF OF THEOREM 3.4}

Throughout this section, $\hat{N}_{t}$ and $\tau_{h}$ are defined by (13) and (14), and the goal is to prove Theorem 3.4. It suffices to prove this theorem for each fixed $0<\theta<\infty$, and there is no loss of generality in supposing that $\theta=1$. With this simplification, $F$ is written for $F_{1}$, and probability is denoted by $P_{N}$, instead of $P_{N, 1}$.

To determine the proper scaling, consider the behavior of $\rho(\omega)$ for small $\omega$. From L'Hospital's Rule, or first principles, it is easily seen that $\rho(\omega) \sim 12 / \omega^{2}$ as $\omega \downarrow 0$. So, if $\hat{\theta}_{t}$ is to remain bounded and $K_{t}$ and $\rho\left(t \hat{\theta}_{t}\right)$ are to be of the same order of magnitude, then $N t$ must be of order $1 / t^{2}$, and it is natural to consider times of the form,

$$
t=N^{-\frac{1}{3}} S,
$$

where $0<s<\infty$; $s$ and $t$ are related by (16) throughout this section. This relation is slightly simpler than using $F^{-1}\left(N^{-\frac{1}{3}} S\right)$ and nearly equivalent since $F(t)=t+O\left(t^{2}\right)$ as $t \downarrow 0$. Let

$$
\begin{gathered}
K_{N}^{o}(s)=N^{-\frac{1}{3}}\left[K_{t}-N\left(1-e^{-t}\right)\right], \\
K_{N}(s)=N^{-\frac{1}{3}}\left[K_{t}-N t\right], \\
H_{N}(s)=3\left[t K_{t}-2\left(\beta+S_{t}\right)\right],
\end{gathered}
$$

and

$$
\Theta_{N}(s)=\hat{\theta}_{t} .
$$

For example, $K_{N}(s)=N^{-\frac{1}{3}}\left[K\left(N^{-\frac{1}{3}} s\right)-N^{\frac{2}{3}} s\right]$. The asymptotic behavior of these processes is central to the proof. To see why observe that

$$
N^{\frac{1}{3}} \tau_{h}=\inf \left\{s>0: t^{2} K_{t} \hat{\theta}_{t}^{2}>\frac{z^{2}}{h^{2}} t^{2} \hat{\theta}_{t}^{2} \rho\left(t \hat{\theta}_{t}\right)\right\}
$$


in which $s$ and $t$ are related by (16). It is shown below that $t^{2} K_{t} \approx s^{3}$ and $t^{2} \hat{\theta}_{t}^{2} \rho\left(t \hat{\theta}_{t}\right) \approx 12$. This leads to the approximate stopping criterion $s^{3} \Theta_{N}(s)^{2}>12 z^{2} / h^{2}$.

There is a simple relation between $K_{N}$ and $K_{N}^{o}$,

$$
K_{N}(s)-K_{N}^{o}(s)=N^{\frac{2}{3}}\left[1-e^{-t}-t\right] \rightarrow-\frac{1}{2} s^{2}
$$

uniformly on compacts and deterministically in $[0, \infty)$. Let $\mathrm{B}(s), 0 \leq s<\infty$ be a standard Brownian motion, and let

$$
H(s)=3 \int_{0}^{s}(s-2 u) d B(u)-6 \beta+\frac{1}{2} s^{3}
$$

for $0 \leq s<\infty$. Then $H(s), 0 \leq s<\infty$, is a Gaussian process with mean and covariance functions

$$
E[H(s)]=\frac{1}{2} s^{3}-6 \beta
$$

and

$$
\operatorname{Cov}\left[H\left(s_{1}\right), H\left(s_{2}\right)\right]=9 \int_{0}^{s_{1}}\left(s_{1}-2 u\right)\left(s_{2}-2 u\right) d u=3 s_{1}^{3}
$$

for $0 \leq s<\infty$ and $0 \leq s_{1} \leq s_{2}<\infty$. Thus, $H(s), 0 \leq s<\infty$, has the same distribution as $B\left(3 s^{3}\right)-6 \beta+s^{3} / 2,0 \leq s<\infty$.

Proposition 5.1 As $N \rightarrow \infty, K_{N}^{o} \Rightarrow B$ and $H_{N} \Rightarrow H$, both in $D[0, \infty)$, for fixed $h>0$.

Proof. The first assertion is an easy exercise using empirical processes. For using the strong approximation theorems of Komlós, Major, and Tusnáldy [6, Theorem 3], and enlarging the probability space, if necessary, there is a sequence of Brownian bridges, $B_{N}$ say, for which

$$
\sup _{0 \leq t \leq \infty}\left|K_{t}-N\left(1-e^{-t}\right)-\sqrt{N} B_{N}\left(1-e^{-t}\right)\right|=O_{p}[\log (N)]
$$

as $N \rightarrow \infty$. See, for example, Csörgö and Révész [4] for background on the strong approximation theorems. Let $\tilde{B}_{N}(s)=N^{\frac{6}{6}} B_{N}\left(N^{-\frac{1}{3}} s\right), \quad 0 \leq s \leq N^{\frac{1}{3}}$ and $\tilde{B}_{N}(s)=0$ for $s>N^{\frac{1}{3}}$. Then $\tilde{B}_{N}$ converges in distribution to a standard Brownian motion in $C[0, \infty)$, and

$$
\sup _{0 \leq s \leq c}\left|K_{N}^{o}(s)-\tilde{B}_{N}(s)\right|=o_{p}(1)
$$


as $N \rightarrow \infty$ for every $0<c<\infty$, by (19) and well known continuity properties of Brownian motion. This establishes the first assertion. For the second, observe that

$$
S_{t}=\sum_{k=1}^{N} T_{k} 1_{\left\{T_{k} \leq t\right\}}=\int_{0}^{t} u d K_{u}
$$

and, therefore,

$$
\begin{aligned}
H_{N}(s) & =3 \int_{0}^{t}(t-2 u) d K_{u}-6 \beta \\
& =3 \int_{0}^{s}(s-2 u) d K_{N}(u)-6 \beta
\end{aligned}
$$

The second assertion then follows from (19), (20), (21), and a transparent integration by parts.

Some detailed information about the distribution of $\hat{\theta}_{t}$ is required for the proof of Theorem 3.4.

Lemma 5.3 Let $\epsilon=\min [\alpha / \beta, 1 / 2]$. Then there is a constant $C$ for which

$$
P_{N}\left\{\hat{\theta}_{t}<\epsilon\right\} \leq \frac{C}{N t^{3}}
$$

for $0<t \leq 1$.

Proof. Let $\mu(\omega)$ denote the mean of $f_{\omega}$ in (11),

$$
\mu(\omega)=-\psi^{\prime}(\omega)=\frac{1}{\omega}-\frac{1}{e^{\omega}-1}
$$

and recall that the variance is $\psi(\omega)$. Then the penalized likelihood equation (12) may be written as

$$
t K_{t} \mu\left(t \hat{\theta}_{t}\right)+\frac{\alpha}{\hat{\theta}_{t}}=\beta+S_{t} .
$$

If $\hat{\theta}_{t}<\epsilon$, then the left side exceeds $\beta$, so that $S_{t}>0, K_{t}>0$, and $t K_{t}[\mu(t \epsilon)-\mu(t)] \leq S_{t}-t K_{t} \mu(t)$. So,

$$
\begin{aligned}
P_{N}\left\{\hat{\theta}_{t}<\epsilon\right\} & \leq \int_{\left\{K_{t}>0\right\}} P_{N}\left\{\left|S_{t}-t K_{t} \mu(t)\right| \geq t K_{t}[\mu(t \epsilon)-\mu(t)] \mid K_{t}\right\} d P_{N} \\
& \leq \int_{\left\{K_{t}>0\right\}} \frac{\psi^{\prime \prime}(t)}{K_{t}[\mu(t \epsilon)-\mu(t) d y]^{2}} d P_{N},
\end{aligned}
$$


using Chebyshev's Inequality on the conditional probability. Now that $\psi^{\prime \prime}(t) \leq 1 / 12$ and that there is a constant $0<C_{0}<\infty$ for which $[\mu(t \epsilon)-\mu(t)] \geq t / C_{0}$ for $0<t \leq 1$. So,

$$
P_{N}\left\{\hat{\theta}_{t}<\epsilon\right\} \leq \frac{C_{0}^{2}}{12 t^{2}} \int_{\left\{K_{t}>0\right\}} \frac{1}{K_{t}} d P_{N} \leq \frac{C_{0}^{2}}{6 N t^{2} F(t)} \leq \frac{C_{0}^{2}}{N t^{3}}
$$

for $0<t \leq 1$.

The asymptotic distribution of $\hat{\theta}_{t}$ is determined next. For this, let

$$
G_{r}(x, y)=\frac{1}{r}\left[1+\frac{2}{e^{r x}-1}-\frac{2}{r x}\right]-\frac{y}{x}
$$

for $0<x, y<\infty$. Then the penalized likelihood equation (12) may be written as

$$
G_{t}\left(\hat{\theta}_{t}, Y_{t}\right)=Z_{t}
$$

where

$$
\begin{gathered}
Y_{t}=2 \alpha / t^{2} K_{t}, \\
Z_{t}=\left(t K_{t}-2 \beta-2 S_{t}\right) / t^{2} K_{t} .
\end{gathered}
$$

The following properties of $G_{r}$ are needed.

Lemma 5.4 For each $r>0$ and $y>0, G_{r}(x, y)$ is a strictly increasing function of $x$. Moreover,

$$
\lim _{r \downarrow 0} G_{r}(x, y)=\frac{1}{6} x-\frac{y}{x}
$$

uniformly on compacts in $0<x, y<\infty$.

Proof. The first assertion follows from differentiation or by observing that (12) is the likelihood equation for a sample from an exponential family when $\alpha=\beta=0$. The second assertion follows from a Taylor series expansion of $e^{r x}$ about $r=0$ and some simple algebra.

Lemma 5.5 The equation $G_{r}(x, y)=z$ has a unique positive solution $x=g_{r}(y, z)$ for all $0<y<\infty$ and $-\infty<z<1 / r$, and

$$
\lim _{r \downarrow 0} g_{r}(y, z)=3 z+\sqrt{9 z^{2}+6 y}
$$

uniformly on compacts in $-\infty<z<\infty$ and $0<y<\infty$. 
Proof. As $x$ increases from 0 to $\infty$. $G_{r}(x, y)$ increases from $-\infty$ to $1 / r$. So, existence and uniqueness of $g_{r}(y, z)$ are clear. To show uniform convergence on compacts, it suffices to show that if $y_{r} \rightarrow y \in(0, \infty)$ and $z_{r} \rightarrow z \in(-\infty, \infty) \quad$ as $\quad r \downarrow 0, \quad$ then $\quad g_{r}\left(y_{r}, z_{r}\right) \rightarrow 3 z+\sqrt{9 z^{2}+6 y}$. Let $x_{r}=g_{r}\left(y_{r}, z_{r}\right)$. Then $x_{r}$ remains bounded away from 0 and $\infty$. For if $x_{r_{k}} \rightarrow 0$ or $\infty$ for some sequence $r_{k} \rightarrow 0$, then $G_{r}\left(x_{r}, y_{r}\right) \rightarrow-\infty$ or $\infty$, which is impossible since $G_{r}\left(x_{r}, y_{r}\right)=z_{r} \rightarrow z$. So $x_{r}=g_{r}\left(y_{r}, z_{r}\right), 0<r \leq 1$, are relatively compact in $(0, \infty)$. If $x$ is any limit point of $x_{r}$ as $r \downarrow 0$, say $x_{r_{k}} \rightarrow x$ as $k \rightarrow \infty$, then $z=\lim _{k \rightarrow \infty} G_{r_{k}}\left(x_{r_{k}}, y_{r_{k}}\right)=x / 6-y / x$ and, therefore, $x=3 z+\sqrt{9 z^{2}+6 y}$. That is, $3 z+\sqrt{9 z^{2}+6 y}$ is the only limit point of $x_{r}$ as $r \downarrow 0$, say $x_{r_{k}} \rightarrow x$ as $k \rightarrow \infty$, then $z=\lim _{k \rightarrow \infty} G_{r_{k}}\left(x_{r_{k}}, y_{r_{k}}\right)$ $=x / 6-y / x$ and, therefore, $x=3 z+\sqrt{9 z^{2}+6 y}$. That is, $3 z+\sqrt{9 z^{2}+6 y}$ is the only limit point of $x_{r}$ as $r \downarrow 0$. The lemma follows.

In the next result, let

$$
\Theta(s)=\frac{H(s)+\sqrt{H(s)^{2}+12 \alpha s^{3}}}{s^{3}},
$$

and $\Theta(0)=\alpha / \beta$. Observe that $\Theta(s), 0 \leq s<\infty$ is a continuous process. Further, as $s \rightarrow \infty, H(s) / s^{3} \rightarrow 1 / 2$ w.p.1, so that

$$
\left|\Theta(s)-2 \frac{H(s)}{s^{3}}\right|=O\left(s^{-3}\right) w \cdot p .1,
$$

by a simple expansion of the square root function. In particular, $\lim _{s \rightarrow \infty}$ $\Theta(s)=1$ w.p.1.

Proposition 5.2 If $\Theta_{N}$ and $\Theta$ are defined as in (17) and (22), $\Theta_{N} \Rightarrow \Theta$ in $D[0, \infty)$ as $N \rightarrow \infty$.

Proof. It is first shown that $\left[t^{2} K_{t}, H_{N}(s)\right] \Rightarrow\left[s^{3}, H(s)\right]$ in $D^{2}[0, \infty)$, using obvious shorthand notation. For this fix a constant $0<c<\infty$. By the Law of Large Numbers $t^{2} K_{t} \rightarrow s^{3}$ in probability for each $0<s<\infty$. Then, by the monotonicity of $K_{t}$ and the continuity of the limit, $\sup _{0 \leq s \leq c}\left|t^{2} K_{t}, H_{N}(s)\right| \Rightarrow\left[s^{3}, H(s)\right]$ in $D^{2}[0, c]$. See Billingsley [2, p.27]. By Skorohod's Theorem, there is a probability space on which are defined processes $\tilde{K}_{t}, \tilde{H}_{N}$, and $\tilde{H}$ for which $\left[t^{2} \tilde{K}_{t}, \tilde{H}_{N}(s)\right]$ has the same distribution as $\left[t^{2} K_{t}, H_{N}(s)\right]$ for each $N, \tilde{H}$ has the same distribution as $H$, and

$$
\lim _{N \rightarrow \infty} \sup _{0 \leq s \leq c}\left[\left|t^{2} \tilde{K}_{t}-s^{3}\right|+\left|\tilde{H}_{N}(s)-\tilde{H}(s)\right|\right]=0 \text { w.p. } 1,
$$


using the fact that convergence in $D[0, c]$ to a convergence function implies uniform convergence. See Billingsley [2, p.112]. Define $\tilde{\Theta}_{N}$ and $\tilde{\Theta}$ by (16) and (21) with $H_{N}$ and $H$ replaced by $\tilde{H}_{N}$ and $\tilde{H}$. Then

$$
\lim _{N \rightarrow \infty} \sup _{0 \leq s \leq c}\left|\tilde{\Theta}_{N}(s)-\tilde{\Theta}(s)\right|=0,
$$

by Lemmas 5.4 and 5.5. The result follows.

Proof of Theorem 3.4: A first passage time is a functional of the form $\inf \{t>0: x(t)>c\} \leq \infty$, defined for $x \in D[0, \infty)$. For example,

$$
\eta_{h}=\inf \left\{s>0: s^{\frac{3}{2}} \Theta(s)>2 \sqrt{3} \frac{z}{h}\right\}
$$

is a first passage time for the process $X$, defined by $X(s)=s^{\frac{3}{2}} \Theta(s)$ for $0 \leq s<\infty$. It is easily seen that any first passage time is continuous almost everywhere with respect to the distribution of $X$ and it then follows from Propositions 5.1 and 5.2 that

$$
\left[N^{\frac{1}{3}} \tau_{h}, \tau_{h}^{2} K\left(\tau_{h}\right), \Theta_{N}\right] \Rightarrow\left(\eta_{h}, \eta_{h}^{3}, \Theta\right)
$$

in $\Re^{2} \times D[0, \infty)$ and, therefore, that

$$
\frac{N}{\hat{N}_{\tau_{h}}}=\frac{N \tau_{h}^{3}}{\tau_{h}^{2} K\left(\tau_{h}\right)} \times \frac{1-e^{-\tau_{h} \hat{\theta}_{\tau_{h}}}}{\tau_{h}} \Rightarrow \Theta\left(\eta_{h}\right) .
$$

Next, since

$$
P_{N}\left\{\left|\hat{N}_{\tau_{h}}-N\right| \leq h \hat{N}_{\tau_{h}}\right\}=P_{N}\left\{\left|\frac{N}{\hat{N}_{\tau_{h}}}-1\right| \leq h\right\},
$$

it follows that

$$
\lim _{N \rightarrow \infty} P_{N}\left\{\left|\hat{N}_{\tau_{h}}-N\right| \leq h \hat{N}_{\tau_{h}}\right\}=P\left\{\left|\Theta\left(\eta_{h}\right)-1\right| \leq h\right\}
$$

for fixed $h>0$. So, to establish (2), it suffices to show that

$$
\lim _{h \downarrow 0} P\left\{\left|\Theta\left(\eta_{h}\right)-1\right| \leq h\right\}=\gamma .
$$

It is clear that $\lim _{h \downarrow 0} \eta_{h}=\infty$ and that $\lim _{s \rightarrow \infty} \Theta(s)=1$, both w.p.l. From sample continuity, it is also clear that $\eta_{h}^{3 / 2} \Theta\left(\eta_{h}\right)=2 \sqrt{3} z / h$ and, therefore, that $\lim _{h \downarrow 0} h \eta_{h}^{3 / 2}=2 \sqrt{3} z$, again both w.p.l. Let

$$
W_{h}(r)=\frac{r^{\frac{3}{2}}}{h}\left[\Theta\left(\frac{r}{h^{\frac{2}{3}}}\right)-1\right] .
$$


Then $W_{h}(r)$ is asymptotically normal with mean 0 and variance 12 as $h \downarrow 0$, by (23), and

$$
P\left\{\left|\Theta\left(\eta_{h}\right)-1\right| \leq h\right\}=P\left\{\left|W_{h}\left(h^{\frac{2}{3}} \eta_{h}\right)\right| \leq h \eta_{h}^{\frac{3}{2}}\right\} .
$$

From (22) and rescaling properties of Brownian motion, it is easily seen that $W_{h}(r), 0<h \leq 1$, are uniformly continuous in probability at any given $r_{0}>0$. It then follows from Anscombe's Theorem that

$$
\lim _{h \downarrow 0} P\left\{\left|W_{h}\left(h^{\frac{2}{3}} \eta_{h}\right)\right| \leq h \eta_{h}^{\frac{3}{2}}\right\}=2 \Phi(z)-1=\gamma,
$$

establishing (2). See, for example, Woodroofe [10, p.10] for suitable version of Anscombe's Theorem.

For (15), we have

$$
\lim _{h \downarrow 0} \lim _{N \rightarrow \infty} P_{N}\left\{\left|h^{\frac{2}{3}} N^{\frac{1}{3}} \tau_{h}-\left(12 z^{2}\right)^{\frac{1}{3}}\right| \geq \epsilon\right\}=\lim _{h \downarrow 0} P\left\{\left|h^{\frac{2}{3}} \eta_{h}-\left(12 z^{2}\right)^{\frac{1}{3}}\right| \geq \epsilon\right\}=0
$$

for all $\epsilon>0$, as in the proof of (9), and the issue becomes the uniform integrability of $h^{2 / 3} N^{1 / 3} F\left(\tau_{h}\right)$. For this it suffices to show that

$$
\lim _{b \rightarrow \infty} \sup _{0<h \leq 1, N \geq 1} \int_{b}^{\infty} P_{N}\left\{h^{\frac{2}{3}} N^{\frac{1}{3}} F\left(\tau_{h}\right)>u\right\} d u=0 .
$$

Let $u=h^{2 / 3} N^{1 / 3} t$ and $b=h^{2 / 3} N^{1 / 3} a$. Then the integral on the right is

$$
h^{\frac{2}{3}} N^{\frac{1}{3}} \int_{a}^{1} P_{N}\left\{F\left(\tau_{h}\right)>t\right\} d t \leq I+I I
$$

where

$$
\begin{gathered}
I=h^{\frac{2}{3}} N^{\frac{1}{3}} \int_{a}^{1} P_{N}\left\{\hat{\theta}_{t}<\epsilon\right\} d t \\
I I=h^{\frac{2}{3}} N^{\frac{1}{3}} \int_{a}^{1} P_{N}\left\{\tau_{h}>t, \hat{\theta}_{t} \geq \epsilon\right\} d t \leq h^{\frac{2}{3}} N^{\frac{1}{3}} \int_{a}^{1} P_{N}\left\{K_{t} \leq \frac{z^{2}}{h^{2}} \rho(t \epsilon)\right\} d t,
\end{gathered}
$$

and $\epsilon=\min [\alpha / \beta, 1 / 2]$, as in Lemma 5.3. By Lemma 5.3, there is a constant $C$ for which

$$
I \leq h^{\frac{2}{3}} N^{\frac{1}{3}} \int_{a}^{1} \frac{C}{N t^{3}} d t \leq h^{\frac{2}{3}} N^{-\frac{2}{3}} \frac{C}{2 a^{2}}=\frac{h^{2}}{2 b^{2}} .
$$


Next, there is another constant, also called $C$, for which $\rho(\omega) \leq C / \omega^{2}$ for all $\omega$. So,

$$
\begin{aligned}
P_{N}\left\{K_{t} \leq \frac{z^{2}}{h^{2}} \rho(t \epsilon)\right\} & \leq P_{N}\left\{\frac{1}{\left(K_{t}+1\right)\left(K_{t}+2\right)}>\left(\frac{h^{2}}{z^{2} \rho(\epsilon t)^{2}+2 h^{2}}\right)^{2}\right\} \\
& \leq\left(\frac{z^{2} \rho(\epsilon t)^{2}+2 h^{2}}{h^{2}}\right)^{2} \frac{1}{N^{2} F(t)^{2}} \\
& \leq \frac{8 z^{4}}{N^{2} h^{4} t^{2}} \rho(\epsilon t)^{2}+\frac{32}{N^{2} t^{2}} \\
& \leq \frac{8 C^{2} z^{4}}{N^{2} \epsilon^{4} h^{4} t^{6}}+\frac{32}{N^{2} t^{2}}
\end{aligned}
$$

and, therefore,

$$
I I \leq h^{\frac{2}{3}} N^{\frac{1}{3}} \int_{a}^{1}\left[\frac{8 z^{4}}{N^{2} \epsilon^{4} h^{4} t^{6}}+\frac{32}{N^{2} t^{2}}\right] d t \leq \frac{2 C^{2} z^{4}}{b^{5}}+\frac{32 h^{\frac{4}{3}}}{N b} .
$$

Uniform integrability follows from the bounds on $I$ and $I I$, completing the proof of the Theorem.

\section{ACKNOWLEDGMENTS}

The research of the second author was supported by the National Science Council of Taiwan and the National Science Foundation; that of the third was supported by the U.S. Army.

\section{REFERENCES}

1. D. Bellout. Order Restricted Estimation of a Distribution with Censored Data and Application to a Stopping Problem. Ph.D. Thesis, Statistics Department, University of Michigan, 1987.

2. P. Billingsley. Convergence of Probability Measures. Wiley, New York, 1968.

3. Y.S. Chow and H. Robbins. Asymptotic theory of fixed width confidence intervals for the mean. Ann. Math. Statist., 36, 457-462, 1965.

4. M. Csörgó and P. Révész, Strong Approximation in Probability and Statistics. Academic Press, New York, 1981. 
5. S.C. Dalal and C.L. Mallows. When should one stop testing software. J. Amer. Statist. Assn., 83, 872-879, 1988.

6. J. Komlós, P. Major, and G. Tusnáldy. An approximation of partial sums of independent RV'-s and the sample DF. I. Z. Wahr. ver. Geb., 32, 111-131, 1975.

7. M. Kramer and N. Starr, N. Discovery rates for a search in which sampling is proportional to size. Adv. in Appl. Math., 8, 222-236, 1987.

8. N. Starr. Optimal and adaptive stopping based on capture times. J. Appl. Probab., 11, 294-301, 1974.

9. Y. Vardi. On a stopping time of starr and its use in estimating the number of transmission sources. J. Appl. Probab., 17, 235-242, 1980.

10. M. Woodroofe. Nonlinear renewal theory in sequential analysis. IMS, Philadelphia, PA, 1982.

Received July 1999

Revised December 2000

Recommended by T.N. Sriram 
